\title{
Effect of survey instrument on participation in a follow-up study: a randomization study of a mailed questionnaire versus a computer-assisted telephone interview
}

\author{
Carissa M Rocheleau ${ }^{1,2^{*}}$, Paul A Romitti ${ }^{1}$, Stacey Hockett Sherlock' ${ }^{1}$, Wayne T Sanderson ${ }^{3}$, \\ Erin M Bell ${ }^{4}$ and Charlotte Druschel ${ }^{4,5}$
}

\begin{abstract}
Background: Many epidemiological and public health surveys report increasing difficulty obtaining high participation rates. We conducted a pilot follow-up study to determine whether a mailed or telephone survey would better facilitate data collection in a subset of respondents to an earlier telephone survey conducted as part of the National Birth Defects Prevention Study.

Methods: We randomly assigned 392 eligible mothers to receive a self-administered, mailed questionnaire (MQ) or a computer-assisted telephone interview (CATI) using similar recruitment protocols. If mothers gave permission to contact the fathers, fathers were recruited to complete the same instrument (MQ or CATI) as mothers.

Results: Mothers contacted for the MQ, within all demographic strata examined, were more likely to participate than those contacted for the CATI (86.6\% vs. $70.6 \%)$. The median response time for mothers completing the MQ was 17 days, compared to 29 days for mothers completing the CATI. Mothers completing the MQ also required fewer reminder calls or letters to finish participation versus those assigned to the CATI (median 3 versus 6), though they were less likely to give permission to contact the father (75.0\% vs. 85.8\%). Fathers contacted for the MQ, however, had higher participation compared to fathers contacted for the CATI ( $85.2 \%$ vs. 54.5\%). Fathers recruited to the MQ also had a shorter response time (median 17 days) and required fewer reminder calls and letters (median 3 reminders) than those completing the CATI (medians 28 days and 6 reminders).

Conclusions: We concluded that offering a MQ substantially improved participation rates and reduced recruitment effort compared to a CATI in this study. While a CATI has the advantage of being able to clarify answers to complex questions or eligibility requirements, our experience suggests that a MQ might be a good survey option for some studies.
\end{abstract}

\section{Background}

Survey research faces two primary challenges: reaching potential subjects, and having potential subjects agree to participate. In the face of rapid societal change, survey methods must constantly be reexamined: new technology, security fears, and laws regarding communications

\footnotetext{
* Correspondence: crocheleau@cdc.gov

'Department of Epidemiology, College of Public Health, The University of lowa, lowa City, IA 52242, USA

${ }^{2}$ National Institute for Occupational Safety and Health, Cincinnati, OH 45226, USA

Full list of author information is available at the end of the article
}

technology may drastically change survey research [1-5]. Survey researchers must therefore adapt their techniques to changing circumstances in order to collect valid and reliable information about a representative sample of individuals.

Though once considered a major breakthrough in survey research, the utility of computer-assisted telephone interviews (CATIs) are challenged by new telephone technology and increasing use of wireless (cellular) telephones instead of (or in addition to) landlines. Caller ID and answering machines/voicemail have made it easier 
for people to screen their calls, making it more difficult for study staff to reach potential participants by telephone [4]. While methods for telephone sampling of landlines are well-established [6,7], sampling wireless numbers faces numerous challenges: numbers are portable, and may not be representative of a geographic region; there are no comprehensive public lists of wireless telephone numbers; federal laws prohibit use of automated dialers when calling wireless telephones; households with wireless telephones and landlines overlap; subjects generally pay for wireless minutes used; and subjects may be in public places when researchers call them, raising privacy considerations $[2,4,5]$.

As wireless telephone usage increases, challenges to telephone-based surveys become more pronounced. Between 2003 and 2009, the number of U.S. households with wireless-only telephones increased from $6.7 \%$ to $24.5 \%[8,9]$. Almost half of young adults, aged 25-29, live in wireless-only households [9]. Urban areas and households living in poverty also have a higher proportion of wireless-only households [8,9]. Demographic differences between people living in wireless-only versus landline households could cause bias in telephone surveys. Target populations with a low prevalence of landlines (e.g. young, urban adults) may also be hard for researchers to access in telephone surveys $[2,10]$.

Given these increasing challenges to telephone surveys, some researchers are reevaluating the utility of mailed questionnaires (MQs). Addresses are often easier to obtain than telephone numbers and are geographically defined. MQs can be answered at a participant's convenience. Several studies conducted from the 1970s through 1990s found that response rates for mailed questionnaires were lower than for telephone surveys [11-13]. Due to changing trends in telephone usage and declining response rates $[4,14]$, these studies may be less relevant to current survey research. A more recent randomized study of survey instruments showed improved participation rates for those who received a MQ versus a CATI [15]. In that study, the difference was most pronounced for women and younger adults, with women in their 30s being 19\% more likely to complete a MQ than a CATI [15].

In comparing instruments, however, it is important to separate the effects of the survey mode from the effects of the recruitment methods. To determine the best survey mode for participation in a follow-up study to a large, multisite population-based study, we conducted a pilot study in which families were randomized to be recruited to complete either a mailed questionnaire (MQ) or CATI using similar recruitment protocols. The purpose of this pilot was to determine whether survey instrument (MQ or CATI) impacted participation rates in a subset of respondents to a previous telephone survey. The pilot also aimed to compare the response rates of mothers and fathers using both survey instruments, and to investigate the representativeness of participants for each instrument.

\section{Methods}

\section{National birth defects prevention study}

Mothers of case or control infants who had completed participation in the National Birth Defects Prevention Study (NBDPS) were recruited. The NBDPS is an ongoing population-based case-control study of birth defects conducted in 10 states in the US (Arkansas, California, Iowa, Georgia, Massachusetts, New Jersey, New York, North Carolina, Texas, and Utah); details have been described elsewhere [16]. The NBDPS protocols are approved by the Human Subjects Review Board of the Centers for Disease Control and Prevention and each of the state-based centers. Mothers of cases and nonmalformed controls completed telephone interviews of their medical and reproductive histories, lifestyle factors, and pregnancy characteristics. At the time of the NBDPS interview, contact information (both address and telephone number) was updated for all participants.

\section{Home and occupational exposure to pesticides study}

As a follow-up study to the NBDPS, a sample of participants was contacted and asked to participate in the Home and Occupational Exposure to Pesticides Study (HOEPS). Eligible mothers were those from the Iowa and New York NBDPS centers who had pregnancies ending between January 1, 2004 and December 31, 2005 and who had already completed participation in the NBDPS. All mothers of infants diagnosed with either hypospadias or a heart defect were recruited, as previous studies have suggested an association between pesticides and these defects $[17,18]$, and mothers of these cases were not enrolled in other follow-up studies. We attempted to recruit one control per case. The HOEPS pilot protocol was approved by the Institutional Review Boards at the University of Iowa and the New York State Department of Health.

Recruitment for the HOEPS occurred from June 2007 to June 2008. Because only mothers participate in the NBDPS interview, recruitment for the HOEPS was initiated with mothers; biological fathers were not contacted for the HOEPS unless permission was given by mothers. Eligible case and control mothers were randomly assigned to be recruited with either a MQ or CATI: each mother was assigned a random number using the RAND function in Microsoft ${ }^{\circledR}$ Excel 2003 (Microsoft Corporation, USA), records were ordered by the assigned random number, and the lower and upper halves were assigned to the MQ and CATI instrument arms, respectively. 
Recruitment followed detailed protocols. In order to isolate the effect of survey instrument from the effects of recruitment methods, the recruitment materials and protocols were designed to provide as comparable an experience as possible to those recruited to the MQ and CATI while using mixed-mode recruitment materials (both telephone and mailed). The only differences in the content of the recruitment materials were instructions/materials that were specific to the survey mode. All mailings were sent via the US Postal Service with address service requested. Where the recorded address or telephone number was no longer accurate, we attempted tracing using a combination of online and/or public data (telephone/address directories, driver license registration, and voter registration), patient contact records, and commercial tracing services. If current contact information could not be identified for a mother, the family was excluded.

Each mother whose contact information could be obtained was initially sent a letter notifying her that we were conducting a follow-up study and would soon be sending out a packet of information about the study. Mothers were given an "address and telephone update sheet" and a pre-addressed, stamped return envelope. Regardless of whether the address update was received, an introductory packet was sent 2 weeks later that included financial compensation (\$20 check). For those randomized to the MQ, a questionnaire and pre-addressed stamped envelope were included in the introductory packet. For those randomized to the CATI, a form indicating preferred times and days to call was enclosed as well as a pre-addressed stamped return envelope; mothers were also given a call-in telephone number if they wished to schedule the interview.

Reminders began after 1 week for those randomized to the CATI and after 2 weeks for those randomized to the MQ (to allow adequate time to review, complete, and return the questionnaire). We attempted up to 3 reminder cycles, two weeks apart, consisting of a telephone reminder followed by a mailed reminder. Because we hoped to deliver a telephone reminder personally, we attempted calling up to 4 times (morning, afternoon, evening, and weekend) for each telephone reminder; we left a message only once (typically on the last call attempt). If we reached the potential participant during any call attempt, the telephone reminder was considered complete. If we did not hear back from potential participants (after 3 days for those assigned to the CATI or 7 days for those assigned to the MQ, to allow for mailing time), we sent a reminder letter. If no response was received after two weeks, the next reminder cycle began.

During the third (final) cycle, the reminder letter informed potential participants that this would be their final contact. For those assigned to the MQ, a copy of the questionnaire and pre-addressed stamped return envelope were enclosed. Those assigned to the CATI were given a form (indicating preferred times and days to be called) and pre-addressed stamped envelope to return if they still wished to participate; they were also provided with a call-in number. If study staff never had direct contact with a potential participant, the decision letter was sent via certified mail. Those who did not respond to the final decision letter were considered nonrespondents (Figure 1). For parents who indicated their intention to participate (by scheduling a telephone interview, returning the CATI decision form, or requesting that a new questionnaire be mailed), we made additional attempts at contact.

The survey instrument covered the six months prior to conception through the end of pregnancy (B6-T3). The maternal and paternal surveys contained a module on

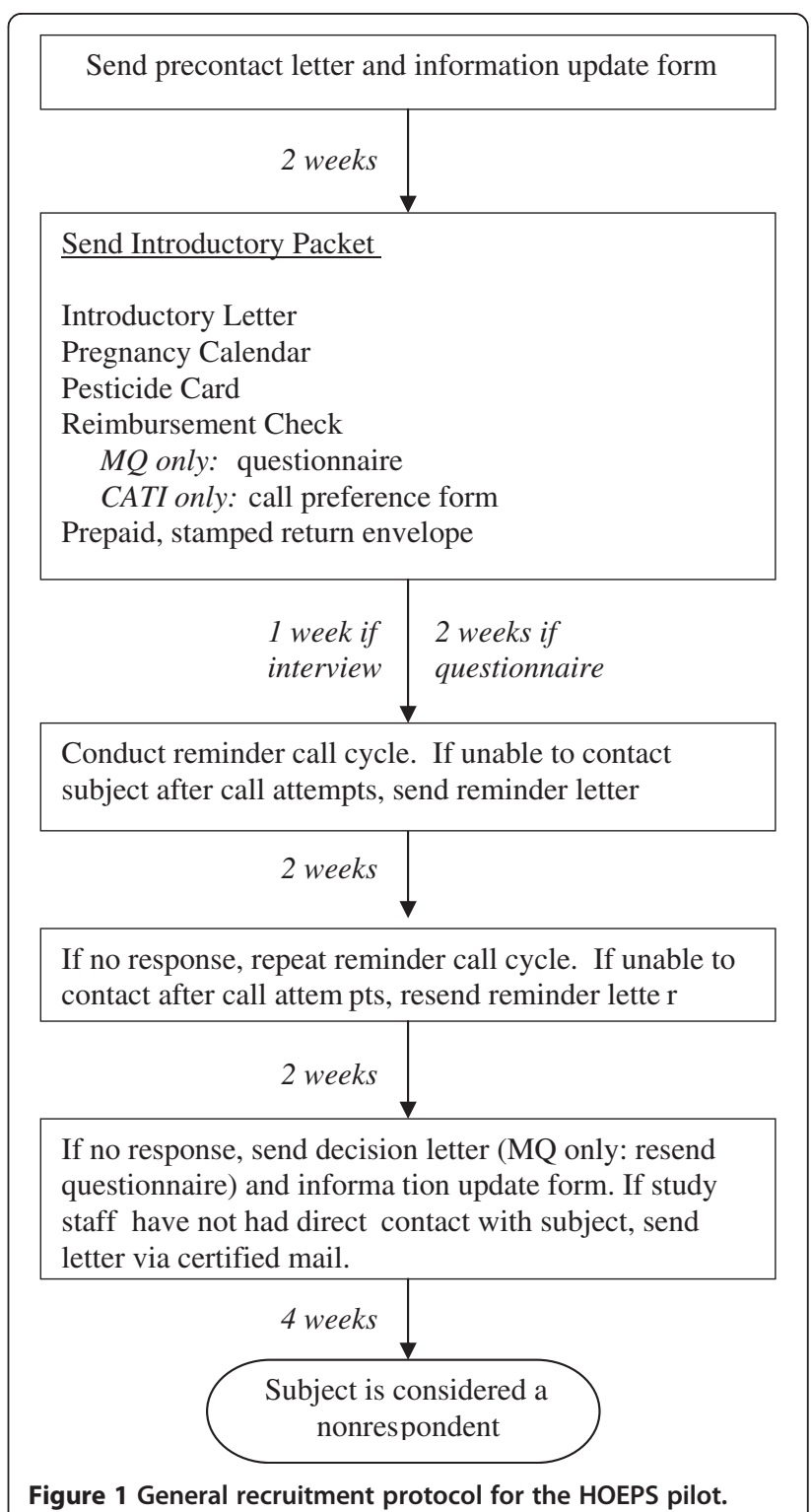


occupational pesticide exposure; the maternal survey also included modules to record residential pesticide treatments, environmental pesticide exposure, and when/if the father resided in the same residence during the survey period. Both the MQ and CATI asked the same questions; however some changes in formatting (such as arranging questions into a table format on the MQ) were made when appropriate for the survey mode. On the last page of the MQ or at the completion of the CATI, mothers were asked for permission to contact the biological father of the index child. If permission was given, contact information for the father was requested (if known). Fathers were assigned to the same instrument (MQ or CATI) as mothers, and were recruited with the same protocol used for mothers.

\section{Statistical analysis}

All analyses were conducted with SAS 9.1.3 (SAS Institute Inc, Cary, NC, USA). Descriptive analyses examined participation rates by case or control status and type of instrument (MQ or CATI); statistical significance $(\mathrm{p}<$ 0.05 ) of the difference in participation rates was calculated using Fisher's Exact test. Bivariable analyses were used to determine demographic characteristics of families participating in the HOEPS, as well as potentially eligible families from the NBDPS, stratified by instrument. For both mothers and fathers, we calculated the median number of days between mailing of the introductory packet and return of a completed questionnaire /completion of an interview and median number of reminders (both telephone and mailed) after mailing the introductory packet, stratified by instrument. The Wilcoxon rank-sum test was used to determine whether the difference in median number of days or number of reminders was statistically significant between instruments $(\mathrm{p}<0.05)$.

\section{Results}

A total of 392 women were eligible for the HOEPS; a current address or telephone number was not identified for 51 (13\%, Figure 1). Contact information could not be identified for more mothers of controls compared to cases $(16.3 \%$ vs. $9.5 \%)$. In total, 341 women were invited to participate in the HOEPS and 268 (68.4\%) completed participation. Among these women, 214 (79.9\%) gave permission to contact the index child's biological father. Valid contact information could not be identified for 5 of these fathers, resulting in 209 fathers who were invited to participate in the HOEPS. Of these, 147 (54.9\% of all fathers) completed participation (Table 1).

Participation rates by instrument and case/control status (Table 2) were calculated only among those who were sent an invitation to participate in HOEPS (i.e., excluding those whose contact information we could not obtain, and fathers we were not given permission to contact). Among the 341 mothers invited to participate in

Table 1 Recruitment status of families eligible for the HOEPS pilot

\begin{tabular}{|c|c|c|c|}
\hline & $N(\%)$ & $N(\%)$ & $N(\%)$ \\
\hline & $\overline{\text { Controls }}$ & Cases $^{a}$ & Total \\
\hline Final Status of Eligible Mothers & $(n=203)$ & $(n=189)$ & $(n=392)$ \\
\hline Excluded: no valid contact information & $33(16.3)$ & $18(9.5)$ & $51(13.0)$ \\
\hline Invited to participate in HOEPS & $170(83.7)$ & $171(90.5)$ & $341(87.0)$ \\
\hline Refused participation & $18(8.9)$ & $15(7.9)$ & $33(8.4)$ \\
\hline Non-response with contact ${ }^{b}$ & $20(9.9)$ & $17(9.0)$ & $37(9.4)$ \\
\hline Non-response without contact ${ }^{\mathrm{b}}$ & $2(1.0)$ & $1(0.5)$ & $3(0.8)$ \\
\hline \multirow[t]{2}{*}{ Completed participation } & $130(64.0)$ & $138(73.0)$ & $268(68.4)$ \\
\hline & Controls & Cases $^{c}$ & Total \\
\hline Final Status of Eligible Fathers & $(n=130)$ & $(n=138)$ & $(n=268)$ \\
\hline Mother did not give permission to contact & $37(28.5)$ & $17(12.3)$ & $214(79.9)$ \\
\hline Excluded: no valid contact information & $3(2.3)$ & $2(1.4)$ & $5(1.9)$ \\
\hline Invited to participate in HOEPS & $90(69.2)$ & $119(86.2)$ & $209(78.0)$ \\
\hline Refused participation & $8(6.2)$ & $4(2.9)$ & $12(4.5)$ \\
\hline Non-response with contact ${ }^{b}$ & $16(12.3)$ & $34(24.6)$ & $50(18.7)$ \\
\hline Non-response without contact ${ }^{b}$ & $0(0)$ & $0(0)$ & $0(0)$ \\
\hline Completed participation & $66(50.8)$ & $81(58.7)$ & $147(54.9)$ \\
\hline
\end{tabular}


Table 2 Participation of parents invited to participate in the HOEPS pilot, by survey instrument and case or control status

\begin{tabular}{|c|c|c|c|c|c|c|c|}
\hline & & MQ & & & CATI & & TOTAL \\
\hline & Cases & Controls & Total & Cases & Controls & Total & Eligible \\
\hline & $\overline{(n=84)}$ & $\overline{(n=87)}$ & $\overline{(n=171)}$ & $\overline{(n=87)}$ & $\overline{(n=83)}$ & $\overline{(n=170)}$ & $(n=341)$ \\
\hline & $N(\%)$ & $N(\%)$ & $N(\%)$ & $N(\%)$ & $N(\%)$ & $N(\%)$ & $N(\%)$ \\
\hline Mother participated $^{a}$ & $73(86.9)$ & $75(86.2)$ & $148(86.6)$ & $65(74.7)$ & $55(66.3)$ & $120(70.6)$ & $268(78.6)$ \\
\hline Permission to contact father ${ }^{a, b}$ & $60(82.2)$ & $51(68.0)$ & $111(75.0)$ & $61(93.9)$ & $42(76.4)$ & $103(85.8)$ & $214(79.9)$ \\
\hline Father participated $d^{\mathrm{a}, \mathrm{c}}$ & $49(83.1)$ & $43(87.8)$ & $92(85.2)$ & $32(53.3)$ & $23(56.1)$ & $55(54.5)$ & $147(70.3)$ \\
\hline Overall father participation ${ }^{\mathrm{a}, \mathrm{d}}$ & $49(58.3)$ & $43(49.4)$ & $92(53.8)$ & $32(36.8)$ & $23(27.7)$ & $55(32.4)$ & $147(43.1)$ \\
\hline
\end{tabular}

a difference between CATI and MQ was significant ( $p$-value $<0.05)$.

${ }^{b}$ the denominator for permission rate is the number of mothers who participated.

c among fathers that project staff were given permission to contact.

d among all fathers potentially eligible for participation at the beginning of HOEPS. Fathers were lost to participation when the mother did not participate, when the mother did not give permission to contact the father, when contact information could not be found for the father, and when the father chose not to participate.

the HOEPS, mothers of case and control infants had similar rates of participation $(80.7 \%$ vs. $76.5 \%$; $\mathrm{p}=0.36$; data not shown).Fathers of the 209 case and control infants who were invited to participate in the HOEPS also had similar participation rates $(68.1 \%$ vs. $73.3 \%$, $\mathrm{p}=0.45$; data not shown). Mothers recruited to the MQ, however, were more likely to participate than those recruited to the CATI ( $86.6 \%$ vs. $70.6 \%$ respectively; $\mathrm{p}<$ 0.001) (Table 2). Among case mothers, participation rates were higher for those assigned to the MQ (86.9\%) versus those assigned to the CATI (74.7\%); there was an even larger difference in participation rates between control mothers recruited to the MQ (86.2\%) versus the CATI (66.3\%) (Table 2).

Cohabitating with the biological father during the six months prior to conception through the end of pregnancy was reported by $86.2 \%$ of participating mothers. Mothers who reported living with the child's biological father during this time period were more likely to give permission to contact the father, compared to mothers who did not live with the father for all of that time period ( $86.2 \%$ vs. $48.65 \%, \mathrm{p}<0.001$; data not shown).

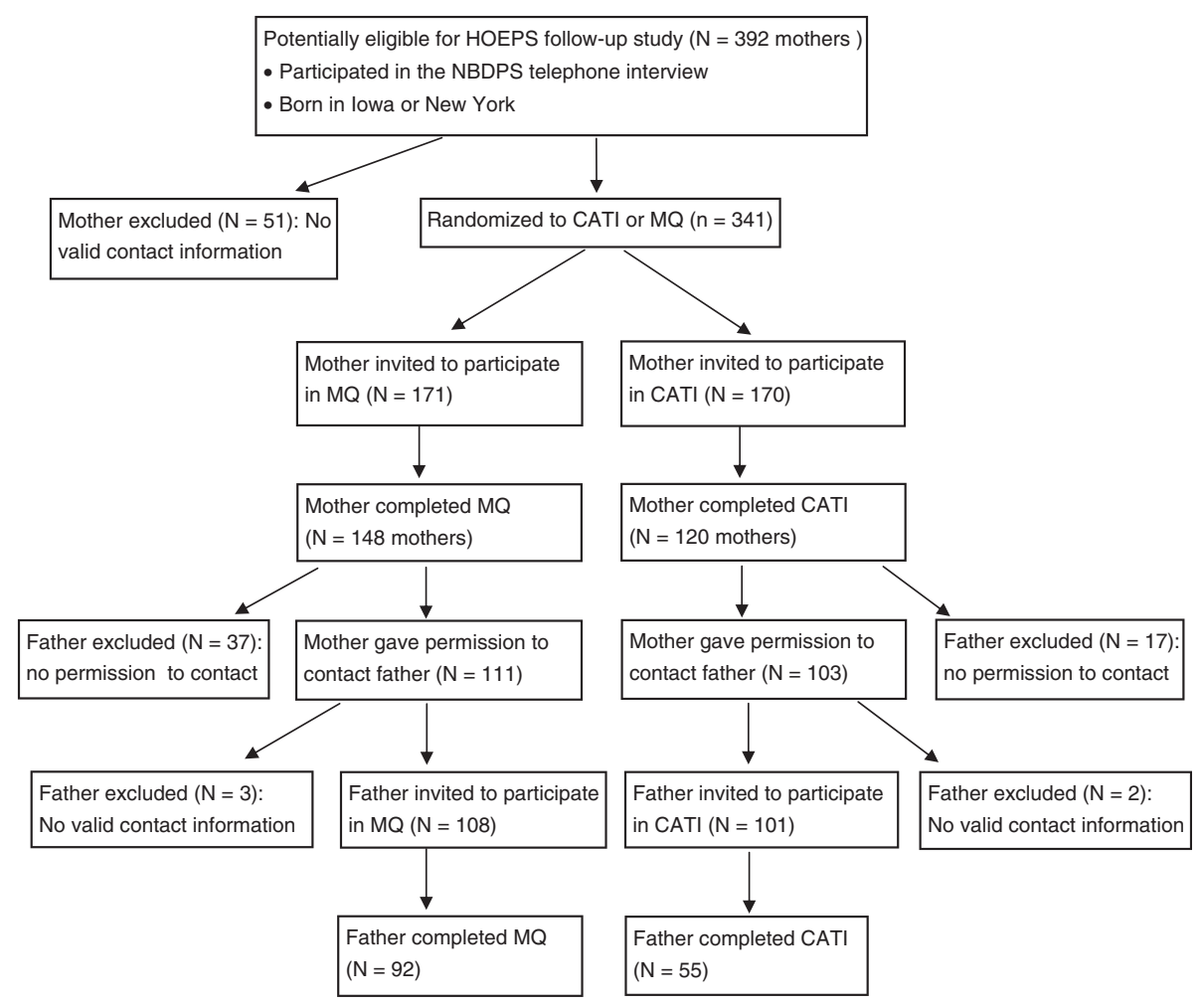

Figure 2 Participation and recruitment of mothers and fathers for the HOEPS pilot. 
Table 3 Participation of parents invited to participate in the HOEPS pilot, by survey instrument and case or control status

\begin{tabular}{|c|c|c|c|c|c|c|}
\hline & \multicolumn{4}{|c|}{ Distribution of demographic characteristics } & \multicolumn{2}{|c|}{ Participation rate } \\
\hline & \multicolumn{2}{|c|}{ Eligible } & \multicolumn{2}{|c|}{ Participated } & & \\
\hline & MQ & CATI & MQ & CATI & MQ & CATI \\
\hline & $(n=195)$ & $(n=197)$ & $(n=148)$ & $(n=120)$ & $(n=148)$ & $(n=120)$ \\
\hline & $N(\%)$ & $N(\%)$ & $N(\%)$ & $N(\%)$ & $\%$ & $\%$ \\
\hline \multicolumn{7}{|l|}{ Case status } \\
\hline Cases & $94(48.2)$ & $95(48.2)$ & $73(49.3)$ & $65(54.2)$ & 77.7 & 68.4 \\
\hline Controls & $101(51.8)$ & $102(51.8)$ & $75(50.7)$ & $55(45.8)$ & 74.3 & 53.9 \\
\hline \multicolumn{7}{|l|}{ Center } \\
\hline lowa & $123(63.1)$ & $123(62.4)$ & $92(62.2)$ & $78(65.0)$ & 74.8 & 63.4 \\
\hline New York & $72(36.9)$ & $74(37.6)$ & $56(37.8)$ & $42(35.0)$ & 77.8 & 56.8 \\
\hline \multicolumn{7}{|l|}{ Mother's age at conception } \\
\hline$<20$ years & $17(8.7)$ & $15(7.6)$ & $10(6.8)$ & $7(5.8)$ & 58.8 & 46.7 \\
\hline 20-24 years & $49(25.1)$ & $45(22.8)$ & $36(24.3)$ & $24(20.0)$ & 73.5 & 53.3 \\
\hline $25-29$ years & $56(28.7)$ & $60(30.5)$ & $42(28.4)$ & $40(33.3)$ & 75.0 & 66.7 \\
\hline 30-34 years & $49(25.1)$ & $57(28.9)$ & $39(26.4)$ & $34(28.3)$ & 79.6 & 59.6 \\
\hline$\geq 35$ years & $24(12.3)$ & $20(10.2)$ & $21(14.2)$ & $15(12.5)$ & 87.5 & 75.0 \\
\hline \multicolumn{7}{|l|}{ Mother's race/ethnicity } \\
\hline White, non-Hispanic & $171(87.7)$ & $176(89.3)$ & $134(90.5)$ & $108(90.0)$ & 78.4 & 61.4 \\
\hline Other race & $21(10.8)$ & $21(10.7)$ & $13(8.8)$ & $12(10.0)$ & 61.9 & 57.1 \\
\hline Missing/Unknown & $3(1.5)$ & $0(0.0)$ & $1(0.7)$ & $0(0.0)$ & $\mathrm{NC}$ & $\mathrm{NC}$ \\
\hline \multicolumn{7}{|l|}{ Mother's education } \\
\hline$<12$ years, or did not complete high school (HS) & $12(6.2)$ & $11(5.6)$ & $6(4.1)$ & $5(4.2)$ & 50.0 & 45.5 \\
\hline 12 years, completed $\mathrm{HS}$, or equivalent & $38(19.5)$ & $43(21.8)$ & $27(18.2)$ & $25(20.8)$ & 71.1 & 58.1 \\
\hline 1-3 years college, or completed technical college & $70(35.9)$ & $68(34.5)$ & $53(35.8)$ & $37(30.8)$ & 75.7 & 54.4 \\
\hline$\geq 4$ years of college; bachelor's degree or higher & $75(38.5)$ & $75(38.1)$ & $62(41.9)$ & $53(44.2)$ & 82.7 & 70.7 \\
\hline \multicolumn{7}{|l|}{ Family's annual income } \\
\hline$<\$ 20,000$ & $36(18.5)$ & $42(21.3)$ & $24(16.2)$ & $22(18.3)$ & 66.7 & 52.4 \\
\hline$\$ 20,000-\$ 39,999$ & $53(27.2)$ & $52(26.4)$ & 39 (26.4) & $32(26.7)$ & 73.6 & 61.5 \\
\hline$\geq \$ 40,000$ & $100(51.3)$ & $98(49.7)$ & $80(54.1)$ & $63(52.5)$ & 80.0 & 64.3 \\
\hline Missing & $6(3.1)$ & $5(2.5)$ & $5(3.4)$ & $3(2.5)$ & 83.3 & 60.0 \\
\hline \multicolumn{7}{|l|}{ Mother smoked } \\
\hline Yes & $56(28.7)$ & $49(24.9)$ & $37(25.0)$ & $21(17.5)$ & 66.1 & 42.9 \\
\hline No & $139(71.3)$ & $147(74.6)$ & $111(75.0)$ & $98(81.7)$ & 79.9 & 66.7 \\
\hline Missing & $0(0.0)$ & $1(0.5)$ & $0(0.0)$ & $1(0.8)$ & $\mathrm{NC}$ & $\mathrm{NC}$ \\
\hline \multicolumn{7}{|l|}{ Parity } \\
\hline Primiparous & $87(44.6)$ & $69(35.0)$ & $67(45.3)$ & $41(34.2)$ & 77.0 & 59.4 \\
\hline Multiparous & $108(55.4)$ & $127(64.5)$ & $81(54.7)$ & $78(65.0)$ & 75.0 & 61.4 \\
\hline Missing & $0(0.0)$ & $1(0.5)$ & $0(0.0)$ & $1(0.8)$ & $\mathrm{NC}$ & $\mathrm{NC}$ \\
\hline \multicolumn{7}{|l|}{ Infant birthweight } \\
\hline$<2500 \mathrm{~g}$ & $21(10.8)$ & $24(12.2)$ & $15(10.1)$ & $9(7.5)$ & 71.4 & 37.5 \\
\hline$\geq 2500 \mathrm{~g}$ & $172(88.2)$ & $170(86.3)$ & $132(89.2)$ & 109 (90.8) & 76.7 & 64.1 \\
\hline Missing & $2(1.0)$ & $3(1.5)$ & $1(0.7)$ & $2(1.7)$ & $\mathrm{NC}$ & $\mathrm{NC}$ \\
\hline
\end{tabular}

NC not calculated. 
Mothers who completed the MQ were less likely to give permission to contact the father compared to mothers who completed the CATI $(75.0 \%$ vs. $85.8 \%$ respectively; $\mathrm{p}=0.03$; Table 2). Fathers invited to complete the $\mathrm{MQ}$, however, had higher participation rates than those invited to complete the CATI ( $85.2 \%$ vs. $54.5 \%$, Table 2 ); consequently more fathers participated with the MQ overall $(n=92)$ compared to the CATI $(n=55$, Figure 2$)$.

The distribution of demographic and pregnancy characteristics (maternal age, race, education, smoking status, parity, family income, and infant birthweight) was similar between mothers who participated in HOEPS and eligible mothers from the NBDPS (Table 3). Within every demographic strata we examined, participation rates were higher for mothers assigned to the MQ versus mothers assigned to the CATI (Table 3).

After receiving their introductory packets, the median time for mothers completing and returning the mailed questionnaire was 17 days; this was significantly $(\mathrm{p}<$ 0.05) shorter than the median time for completing the CATI (29 days, Table 4). Mothers assigned to the MQ also required significantly fewer reminders (median of 3 reminders versus $6, \mathrm{p}<0.05$ ). Fathers participating in the MQ also completed their surveys in less time compared to fathers participating in the CATI (median 28 days vs. 17 days; $\mathrm{p}=0.09$ ), and required fewer reminders (median
3 vs. 6 ; $p<0.001$ ) (Table 4). There was no significant difference between survey modes in time between birth of the index child and completion of the HOEPS (Table 3).

\section{Discussion}

Overall, participation among recruited mothers was good in the HOEPS. Fathers invited to participate in the HOEPS also had high participation rates, unlike a previous study that found women were $10 \%$ more likely to complete an initial survey than men [19]. Fathers were lost to participation, however, when the mother could not be located, refused participation in HOEPS, or did not give permission to contact the father. Information provided from fathers directly may be more accurate than those reported by proxy, however [20-24]. Our results show that obtaining information from fathers, in addition to mothers, is feasible though participation rates may be lower when mothers are 'gatekeepers' to study participation. In studies of reproductive outcomes, mothers are often contacted first: mothers are the sources of information (or permission to medical records) about pregnancy and delivery characteristics, fathers may not be specified on birth records, and biological fathers may not be aware of the birth of the child. Since mothers who resided with the index child's biological father six months before and during the index pregnancy were more likely

Table 4 Response characteristics of participants in HOEPS pilot, by survey instrument and case or control status

\begin{tabular}{|c|c|c|c|}
\hline & Cases Median (IR) & Controls Median (IR) & Total Median (IR) \\
\hline Mothers & $N=138$ & $N=130$ & $N=268$ \\
\hline \multicolumn{4}{|c|}{ Time to completion (days) $^{a}$} \\
\hline CATI & $29.0(15.0-56.0)$ & $29.0(13.0-43.0)$ & $29(14.0-51.5)$ \\
\hline MQ & $20.0(13.0-35.0)$ & $17.0(9.0-39.0)$ & $17(10.0-35.0)$ \\
\hline \multicolumn{4}{|c|}{ Number of reminders ${ }^{a, b}$} \\
\hline CATI & $6.0(4.0-9.0)$ & $6.0(4.0-8.0)$ & $6.0(4.0-8.0)$ \\
\hline MQ & $3.0(2.0-5.0)$ & $3.0(2.0-4.0)$ & $3.0(2.0-4.0)$ \\
\hline \multicolumn{4}{|c|}{ Time elapsed from child's birth to completion of HOEPS (years) } \\
\hline CATI & $2.9(2.2-3.2)$ & $2.6(2.1-3.2)$ & $2.8(2.2-3.2)$ \\
\hline MQ & $2.7(2.3-3.3)$ & $2.6(2.1-3.2)$ & $2.6(2.2-3.3)$ \\
\hline Fathers & $N=81$ & $N=66$ & $N=147$ \\
\hline \multicolumn{4}{|c|}{ Time to completion (days) } \\
\hline CATI & $28.0(14.0-53.5)$ & $21.0(16.0-42.0)$ & $28.0(14.0-49.0)$ \\
\hline MQ & $20.0(13.0-41.0)$ & $16.0(10.0-41.0)$ & $17.0(10.0-41.0)$ \\
\hline \multicolumn{4}{|c|}{ Number of reminders ${ }^{a, b}$} \\
\hline CATI & $6.0(4.0-6.5)$ & $5.0(4.0-7.0)$ & $6.0(4.0-7.0)$ \\
\hline MQ & $3.0(1.0-5.0)$ & $2.0(1.0-5.0)$ & $3.0(1.0-5.0)$ \\
\hline \multicolumn{4}{|c|}{ Time elapsed from child's birth to completion of HOEPS (years) } \\
\hline CATI & $3.1(2.6-3.4)$ & $2.6(2.3-3.5)$ & $3.0(2.4-3.5)$ \\
\hline MQ & $2.9(2.4-3.4)$ & $2.7(2.3-3.2)$ & $2.8(2.3-3.3)$ \\
\hline
\end{tabular}

IR interquartile range.

${ }^{a}$ difference between CATI and MQ was statistically significant $(p<0.05)$.

b This includes reminder letters and calls; a reminder call consists of up to 4 call attempts. Participants who returned the MQ without a reminder, or who called in to schedule/complete an interview after receiving the introductory packet, had 0 reminders. 
to give permission for researchers to contact the father, this could be an important source of selection bias in evaluating paternal exposures in studies of birth outcomes where mothers act as gatekeepers.

Fewer mothers completing the MQ gave permission to contact fathers compared to mothers completing the CATI; this was not explained by underlying differences in the percentages of mothers who reported living with their child's biological father during the entirety of B6-T3 . Mothers who received the MQ had the opportunity to ask fathers if they wanted to be contacted before giving us permission; this may explain why mothers assigned to the MQ tended to give permission less often to contact the father, but the fathers were more likely to participate when contacted. During the CATI, mothers also had an opportunity to clarify what was expected of the father (including what information would be solicited and how long the interview would take). Sending both the maternal and paternal MQs to the mother at the same time might increase paternal participation; mothers could then choose whether or not to pass the paternal MQ to the father immediately if they share a residence.

Because the HOEPS was a follow-up among previous study participants, our population is probably more motivated and the overall participation rates we observed are likely higher than would be expected in a survey within the general population. This structure, however, created an excellent opportunity for comparing participation between instruments: we had good contact information (both telephone and mailing), allowing our recruitment protocols to be similar between both instruments; all families were drawn from the same population of NBDPS participants and randomly assigned to an instrument, removing most potential confounding/bias in a comparison of the instruments; and we had demographic information on eligible non-participants, allowing us to evaluate differences in participation rates between specific subgroups. Our design may have been somewhat biased towards high participation rates among those assigned to the CATI, because participation in the NBDPS requires completing a telephone interview. All those eligible for the HOEPS therefore had telephone access and a demonstrated willingness to provide information over the telephone.

We found that participation rates were higher for both mothers and fathers assigned to the MQ versus the CATI. Participants assigned to the MQ also required fewer reminder call cycles or letters before completing the study versus participants assigned to the CATI. As we expected, the largest impact of survey method on participation rates occurred among control subjects. Case subjects are generally more highly motivated than control subjects, thus we would expect the attractiveness of a survey method to have less weight in motivating participation among cases than controls. In all demographic strata we examined, participation rates were higher for mothers assigned to the MQ versus the CATI. The distribution of participating subjects across demographic groups, however, was similar to the distribution of eligible subjects. This suggests that mothers recruited to the HOEPS are likely representative of NBDPS participants, and that the increased response to the MQ was not attributable to a specific demographic subgroup.

Future studies that evaluate the use of a MQ should consider whether the increased cost of postage and printing for a questionnaire will be offset by savings in recruitment costs and increased participation rates. We found that the MQ saved personnel time (primarily due to fewer reminders) compared to the CATI, while postage and printing costs of the MQ were only slightly increased compared to those for the CATI. Overall, the mailed questionnaire was more cost-effective for this study, though following up on missing or confusing responses to the MQ required considerable time and effort whereas interviewers could immediately probe vague answers during the CATI. Complex surveys soliciting detailed answers may therefore be better suited to a CATI than a MQ.

There are several important limitations to this study. The increased participation rates we observed among those invited to the MQ versus the CATI may not be generalizeable to all groups; our population consisted primarily of the parents of young children who might have difficulty finding quiet time to complete a telephone interview or predicting when they will have free time. A paper questionnaire might be easier for these busy parents to fill out a little at a time when opportunities arise. In this study, we were also unable to compare the impact of survey type on the validity of respondents' answers. Research from the 1970s and 1980s found that responses to MQs were generally equivalent to [25] or more valid than responses to CATIs [24]. A 1979 study of item omission between mailed and telephone surveys found that participants were more likely to answer sensitive questions on a mailed survey [13]. These studies, however, were undertaken in decades past and it may not be accurate to apply these results to current survey research; few similar studies have been conducted recently. We also did not evaluate the impact of offering more than one mode of survey instrument, which has been shown to improve participation rates [26,27]. As internet coverage increases, web-based surveys may also provide a practical and economical alternative.

Despite these limitations, this study provides a valuable comparison of survey instruments. Since subjects were randomized to the MQ or CATI, neither self-selection effects nor confounding is likely to affect the comparison of the two instruments. This is supported by the similar 
distribution of maternal demographic characteristics between mothers assigned to each instrument, as well as the similarities we observed between all those eligible for HOEPS and those who completed participation. We obtained a higher response rate with less recruitment effort using the MQ.

\section{Conclusions}

In a follow-up survey, we randomized families to be recruited to a MQ or a CATI. Both instruments contained the same information and similar recruitment protocols and materials. We obtained higher participation among mothers recruited to complete a MQ versus a CATI. This higher participation rate for the MQ held true in every demographic subgroup we examined. Although mothers who completed the MQ were less likely to give us permission to contact fathers, those fathers we contacted were more likely to participate compared to fathers recruited with the CATI; consequently we had higher overall participation from fathers using the MQ. While we found that it was very time-consuming to follow up on missing or confusing responses to the MQ, we also did not have to devote as much time to sending reminders for the MQ compared to the CATI. The costbenefit ratio of one instrument versus another may vary depending on the target population and complexity of information being sought. Such information may guide other epidemiological studies, particularly those targeting the parents of young children, in deciding which mode of survey administration to implement.

\section{Competing interests}

The authors declare that they have no competing interests.

\section{Authors' contributions}

PAR conceived the study. All authors assisted in designing the survey instruments and/or recruitment protocols for the HOEPS. CMR prepared all IRB documents, assisted in participant recruitment/ data management, and performed data analysis. SHS oversaw recruitment and data collection. All authors contributed to writing and revising this manuscript. All authors read and approved the final manuscript.

\section{Acknowledgements}

The authors would like to thank Kara Grasty and Holly Erschens from The University of lowa for their assistance in recruitment and interviewing participants, and Tanner Wenzel from The University of lowa for designing the recruitment tracking databases and programming the CATI. This work was funded by grants sponsored by the Centers for Disease Control and Prevention (U50CCU713238/ U01DD000492 and U50CCU223184/ U01DD0004870). Dr. Rocheleau also received dissertation support from the Heartland Center for Occupational Safety and Health at the University of lowa, a NIOSH-funded Education and Research Center. No funding body had any role in the design, collection, analysis, and interpretation of data; nor was any funding body involved in the writing of the manuscript or in the decision to submit the manuscript for publication. The findings and conclusions in this report are those of the authors and do not necessarily represent the views of the National Institute for Occupational Safety and Health or Centers for Disease Control and Prevention.

\section{Author details}

'Department of Epidemiology, College of Public Health, The University of lowa, lowa City, IA 52242, USA. ${ }^{2}$ National Institute for Occupational Safety and Health, Cincinnati, OH 45226, USA. ${ }^{3}$ Department of Occupational and Environmental Health, College of Public Health, The University of lowa, lowa City, IA 52242, USA. ${ }^{4}$ Department of Epidemiology and Biostatistics, School of Public Health, State University of New York at Albany, Rensselaer, NY 12144, USA. ${ }^{5}$ New York State Department of Health, Troy, NY 12180, USA.

Received: 29 March 2012 Accepted: 17 July 2012

Published: 31 July 2012

\section{References}

1. Blumberg SJ, Luke JV, Cynamon ML: Telephone coverage and health survey estimates: evaluating the need for concern about wireless substitution. Am J Public Health 2006, 96(5):926-931.

2. Cantor JC, Brownlee S, Zukin C, Boyle JM: Implications of the growing use of wireless telephones for health care opinion polls. Health Serv Res 2009, 44(5 Pt 1):1762-1772.

3. Curtin R, Presser $S$, Singer E: Changes in Telephone Survey Nonresponse over the Past Quarter Century. Public Opin Q 2005, 69(1):87-98.

4. Kempf AM, Remington PL: New challenges for telephone survey research in the twenty-first century. Annu Rev Public Health 2007, 28:113-126.

5. Tourangeau R: Survey research and societal change. Annu Rev Psychol 2004, 55:775-801.

6. Lavrakas PJ: Telephone Survey Methods: Sampling, Selection, and Supervision. Newbury Park, CA: Sage; 1993.

7. Groves RM, Biemer PP, Lyberg LE, Massey JT, Nichlolls WL, Waksberg J (Eds): Telephone Survey Methodology. New York: Wiley; 1988.

8. Blumberg SJ, Luke JV, Davidson G, Davern ME, Yu TC, Soderberg K: Wireless substitution: state-level estimates from the National Health Interview Survey, January-December 2007. Natl Health Stat Rep 2009, 16(14):1-13.

9. Blumberg BS, Luke JV: Wireless Substitution: Early Release of Estimates from the National Health Interview Survey, July-December 2009: National Center for Health Statistics; 2010. Available from: http://www.cdc.gov/nchs/nhis.htm.

10. Bernal R, Silva NN: Home landline telephone coverage and potential bias in epidemiological surveys. Rev Saude Publica 2009, 43(3):421-426.

11. Harris LE, Weinberger M, Tierney WM: Assessing inner-city patients' hospital experiences. A controlled trial of telephone interviews versus mailed surveys. Med Care 1997, 35(1):70-76.

12. O'Toole Bl, Battistutta D, Long A, Crouch K: A comparison of costs and data quality of three health survey methods: mail, telephone and personal home interview. Am J Epidemiol 1986, 124(2):317-328.

13. Siemiatycki J: A comparison of mail, telephone, and home interview strategies for household health surveys. Am J Public Health 1979, 69(3):238-245.

14. Mokdad AH: The Behavioral Risk Factors Surveillance System: past, present, and future. Annu Rev Public Health 2009, 30:43-54.

15. Feveile $\mathrm{H}$, Olsen $\mathrm{O}$, Hogh $\mathrm{A}$ : A randomized trial of mailed questionnaires versus telephone interviews: response patterns in a survey. BMC Med Res Methodol 2007, 7:27.

16. Yoon PW, Rasmussen SA, Lynberg MC, Moore CA, Anderka M, Carmichael SL, Costa P, Druschel C, Hobbs CA, Romitti PA, et al: The National Birth Defects Prevention Study. Public Health Rep 2001, 116(Suppl 1):32-40.

17. Rocheleau CM, Romitti PA, Dennis LK: Pesticides and hypospadias: a metaanalysis. J Pediatr Urol 2009, 5(1):17-24.

18. Loffredo CA, Silbergeld EK, Ferencz C, Zhang J: Association of transposition of the great arteries in infants with maternal exposures to herbicides and rodenticides. Am J Epidemiol 2001, 153(6):529-536.

19. Hensley Alford S, McBride CM, Reid RJ, Larson EB, Baxevanis AD, Brody LC: Participation in Genetic Testing Research Varies by Social Group. Public Health Genomics 2011, 14(2):85-93.

20. Campbell PT, Sloan M, Kreiger N: Utility of proxy versus index respondent information in a population-based case-control study of rapidly fatal cancers. Ann Epidemiol 2007, 17(4):253-257.

21. Fryzek JP, Lipworth LL, Garabrant DH, McLaughlin JK: Comparison of surrogate with self-respondents for occupational factors. J Occup Environ Med 2000, 42(4):424-429.

22. Johnson RA, Mandel JS, Gibson RW, Mandel JH, Bender AP, Gunderson PD, Renier CM: Data on prior pesticide use collected from self- and proxy respondents. Epidemiology 1993, 4(2):157-164.

23. Schnitzer PG, Olshan AF, Savitz DA, Erickson JD: Validity of mother's report of father's occupation in a study of paternal occupation and congenital malformations. Am J Epidemiol 1995, 141(9):872-877. 
24. Siemiatycki J, Campbell S, Richardson L, Aubert D: Quality of response in different population groups in mail and telephone surveys. Am J Epidemiol 1984, 120(2):302-314.

25. Hochstim J: A critical comparison of three strategies of collecting data from households. J Am Stat Assoc 1976, 62:976-989.

26. Brambilla DJ, McKinlay SM: A comparison of responses to mailed questionnaires and telephone interviews in a mixed mode health survey. Am J Epidemiol 1987, 126(5):962-971.

27. Fowler FJ Jr, Gallagher PM, Stringfellow VL, Zaslavsky AM, Thompson JW, Cleary PD: Using telephone interviews to reduce nonresponse bias to mail surveys of health plan members. Med Care 2002, 40(3):190-200.

doi:10.1186/1471-2458-12-579

Cite this article as: Rocheleau et al.: Effect of survey instrument on participation in a follow-up study: a randomization study of a mailed questionnaire versus a computer-assisted telephone interview. BMC Public Health 2012 12:579.

\section{Submit your next manuscript to BioMed Central and take full advantage of:}

- Convenient online submission

- Thorough peer review

- No space constraints or color figure charges

- Immediate publication on acceptance

- Inclusion in PubMed, CAS, Scopus and Google Scholar

- Research which is freely available for redistribution 